\title{
A Highly Efficient Treatment of Rheumatoid Arthritis
}

\author{
Kimihiko 0* \\ Okazaki Medical Clinic, Japan
}

*Corresponding author: Kimihiko Okazaki, Okazaki Medical Clinic, Ukyoku, Kyoto, Japan, Tel: 81-75-314-8123; E-mail: ma13081x@ma1.seikyou.ne.jp

Short Communication

Volume 1 Issue 1

Received Date: July 07, 2017

Published Date: July 17, 2017

DOI: $10.23880 /$ mjccs-16000106

\section{Abstract}

A purely medical, not surgical, treatment for rheumatoid arthritis is demonstrated. In short, it cures the disease via elimination of the cause of the disease, namely, decomposition of combinations of cartilage-specific antibody and cytolytic $\mathrm{T}$ lymphocyte. The latter is accomplished by replacements of cartilage-specific antibody with non-specific antibody. Still the latter is accomplished by accumulation of non-specific antibodies in the patient's body. Still the latter is accomplished by repetitions of intradermal injections with a non-specific antigen preparation.

\section{Introduction}

It is well known that rheumatoid arthritis is an autoimmune disease. As a matter of course, all of patients of this disease should have anti-cartilage antibodies on the surface of cytolytic $\mathrm{T}$ lymphocytes. Few, if any, contemporary physicians seem to take this fact into consideration presumably because most, if not all, of them take it for granted that most, if not all, antibody molecules rigidly adhere to their receptors on cell-surfaces.

On the other hand, approximately five decades ago, a novel concept of existence of equilibrium state among antibody molecules in the vicinity of their receptors on cell-surfaces has been established [1,2]. It follows that every antibody molecule in the vicinity of its receptors keeps repeating attaching to and detaching from the receptors. The reason why not "receptor" but "receptors" is because each antibody molecule may change receptors. It still follows that these replacements of antibody molecules on a certain receptor keep occurring all the time. Naturally, the traditional concept of rigid adhesions of antibody molecules to the receptors is irrelevant.

\section{Conclusion}

In conclusion, progressive destruction of cartilage could be terminated if anti-cartilage antibodies were replaced from the surface of cytolytic T lymphocytes. In order to do so, accumulations of non-specific antibodies in the patients' bodies are necessary. Still in order to do so, repeated intradermal injections with a non-specific antigen preparation are necessary. An example of the latter preparation is Neurotropin, a product of Nippon Pharmaceutical Company (Osaka), consisting of extract of rabbit skin inflamed by inoculation of Vaccinia virus. Numerous successful cases of the above mentioned treatment for rheumatoid arthritis have accumulated and are still accumulating in the author's clinic.

An alternative proposition might be intra-venous infusions of saline solution of gamma-globulin. However, repeated gamma-globulin infusions are dangerous since anti-gamma-globulin antibodies may be produced in the recipients' bodies, which may cause an anaphylactic reaction after a large number of infusions. 


\section{Medical Journal of Clinical Trials \& Case Studies}

\section{References}

1. Andrews P (1965) The gel-filtration behavior of proteins related to their molecular weights over a wide range. Biochem J 96(3): 595-606.
2. Rodewald $\mathrm{R}$ (1976) pH-dependent binding of immunoglobulins to intestinal cells of the neonatal rat. J Cell Biol 71(2): 666-669. 